\title{
Disconnecting a traversable wormhole: Universal quench dynamics in random spin models
}

\author{
Tian-Gang Zhou $\odot,{ }^{1}$ Lei Pan, ${ }^{1}$ Yu Chen, ${ }^{2}$ Pengfei Zhang,,${ }^{3,4, *}$ and Hui Zhai $\oplus^{1, \dagger}$ \\ ${ }^{1}$ Institute for Advanced Study, Tsinghua University, Beijing 100084, China \\ ${ }^{2}$ Graduate School of China Academy of Engineering Physics, Beijing 100193, China \\ ${ }^{3}$ Institute for Quantum Information and Matter, California Institute of Technology, Pasadena, California 91125, USA \\ ${ }^{4}$ Walter Burke Institute for Theoretical Physics, California Institute of Technology, Pasadena, California 91125, USA
}

(Received 1 December 2020; accepted 7 June 2021; published 21 June 2021)

\begin{abstract}
Understanding strongly interacting quantum matter and quantum gravity are both important open issues in theoretical physics, and the holographic duality between quantum field theory and gravity theory nicely brings these two topics together. Nevertheless, direct connections between gravity physics and experimental observations in quantum matter are still rare. Here we utilize the gravity physics picture to understand quench dynamics experimentally observed in a class of random spin models realized in several different quantum systems, where the dynamics of magnetization are measured after the external polarization field is suddenly turned off. Two universal features of the magnetization dynamics, namely, a slow decay described by a stretched exponential function and an oscillatory behavior, are respectively found in different parameter regimes across different systems. This paper addresses the issues of generic conditions under which these two universal features can occur, and we find that a natural answer to this question emerges in the gravity picture. By the holographic duality bridged by a model proposed by Maldacena and Qi, the quench dynamics after suddenly turning off the external polarization field is mapped to disconnecting an eternal traversable wormhole. Our studies show that insight from gravity physics can help unifying different experiments in quantum systems.
\end{abstract}

DOI: 10.1103/PhysRevResearch.3.L022024

\section{INTRODUCTION}

The holographic duality between quantum field theory at boundary and gravity theory in bulk has shed new insights in understanding both quantum matter and gravity [1-3]. For example, on the gravity side, the wormhole in local Einstein gravity is not traversable, and recently mechanisms that can render a wormhole traversable have been studied by coupling quantum fields on boundaries [4-6]. On the quantum matter side, insights from gravity theory can help us understand strongly interacting quantum systems, such as non-Fermi liquids [2,3]. Nevertheless, so far only a few specifically designed quantum models such as the SachdevYe-Kitaev (SYK) model [7-10] have been explicitly shown to possess holographic duality to gravity theory. Such a model is seemingly different from realistic systems in quantum materials and is also hard to be realized by quantum simulations. Therefore, so far little connection between sights from gravity physics and realistic experimental observations in quantum matter has been established through holographic duality,

\footnotetext{
*pengfeizhang.physics@gmail.com

†hzhai@tsinghua.edu.cn
}

Published by the American Physical Society under the terms of the Creative Commons Attribution 4.0 International license. Further distribution of this work must maintain attribution to the author(s) and the published article's title, journal citation, and DOI. except for few known examples such as bounds for viscosity and transport coefficients [11-15].

In this paper we focus on a typical random spin model written as

$$
\hat{H}=\sum_{i<j} J_{i j}\left(\hat{S}_{i}^{x} \hat{S}_{j}^{x}+\hat{S}_{i}^{y} \hat{S}_{j}^{y}+\Delta \hat{S}_{i}^{z} \hat{S}_{j}^{z}\right)-h \sum_{i} \hat{S}_{i}^{x} .
$$

This model has recently been realized by cold molecules in optical lattices [16,17], and NV centers [18], fermions in harmonic traps [19], Rydberg atoms [20], high-spin atoms in optical lattices [21], and by solid-state NMR [22,23]. $J_{i j}$ is random all-to-all spin interaction coefficients, which originates either from dipolar interaction or from the spinexchanging interaction, and the randomness comes from the random locations of spin carriers in these systems. We can denote $J_{i j}=\bar{J} / N+\delta J_{i j}$, where $\bar{J} / N$ is the averaged value of all $J_{i j}$ and $N$ is the number of spins. $\left\langle\delta J_{i j}^{2}\right\rangle=4 J^{2} / N$ and $J$ denotes the typical strength of random spin interactions. $\Delta$ is the anisotropy between the longitudinal and the transverse spin-spin interactions. $h$ is an external polarization field. Among these systems, $\hbar / J$ varies from a few microseconds to a few milliseconds, and the values of $\bar{J} / J$ and $\Delta$ are also different.

Taking the advantages of the controllability of these systems, these realizations enable experimental studies of far-from-equilibrium quantum dynamics, and the quench dynamics in these random spin models have also been reported in a number of recent experiments [16-21]. In the quench dynamics, spins are initially polarized in the transverse direction, say, along $\hat{x}$, by $h$ term in Eq. (1), and then the dynamics of the 
(a)
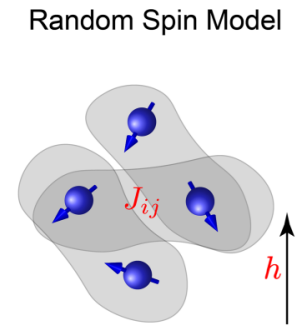

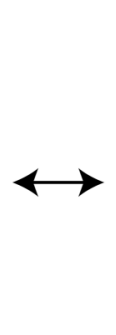

(b)

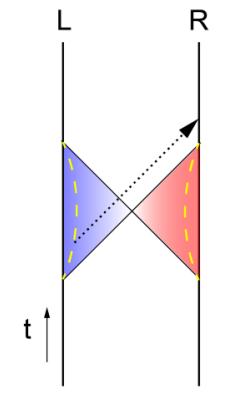

Black Hole (BH)
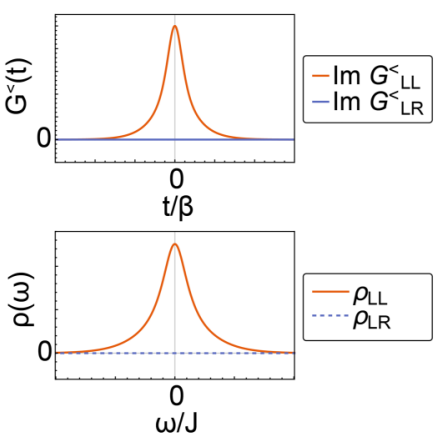

Maldacena-Qi Model

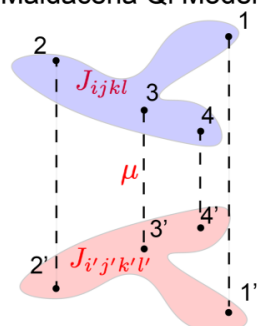

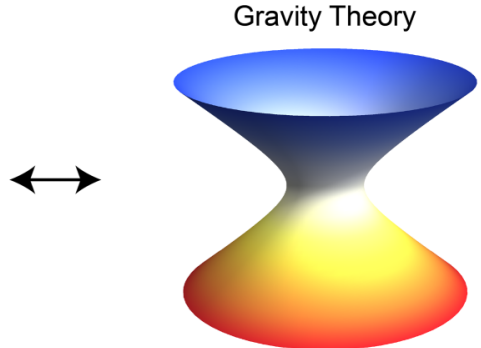

$\mathrm{R}$
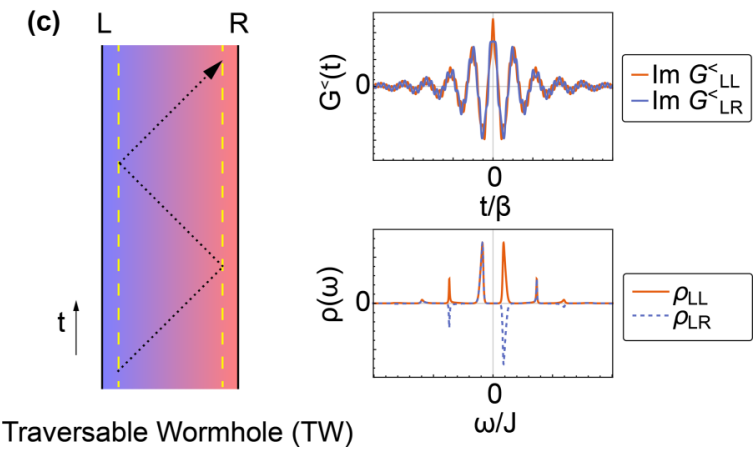

Traversable Wormhole (TW)

FIG. 1. Illustration of models and phases. (a) The connection between the random spin models and the gravity theory bridged by the Maldacena-Qi model. [(b), (c)] Illustration of the black hole (BH) phase and the eternal traversable wormhole (TW) phase in the Maldacena-Qi model and its dual gravity theory. The correspondence between the Penrose diagram in the gravity theory (left) and the lesser Green's function $G^{<}(t)$ as well as the spectral function $\rho(\omega)$ (right) in the quantum theory, are shown for the BH phase (b) and the TW phase (c), respectively. In the Penrose diagrams, the colored regimes are the physical space in the geometry. Two yellow dashed lines represent the physical boundaries of two $\mathrm{AdS}_{2}$ spaces, denoted by the left $(\mathrm{L})$ and the right $(\mathrm{R})$ ones, where the quantum fields are defined. The black dotted lines with an arrow represent the null geodesic. The horizontal axis is the real-time axis.

total magnetization along $\hat{x}$ are experimentally measured after $h$ is suddenly turned off. In some of these experiments where the magnetization decay time due to the single-spin effect is shorter, a spin echo is applied to eliminate the single-spin effect [16-18,21], which ensures that the observed features are all due to spin-spin interactions described by Eq. (1). Two features of the quench spin dynamics have been observed in the time scale from a few to a few tens of $\hbar / J$. They take place in different parameter regimes, but both are observed in different systems and across different parameter regimes. These two features are listed below:

(i) Slow decay of the total magnetization has been found when the magnetization decays to zero at long time, and the time dependence of the total magnetization is identified as a stretched exponential function. This feature has been observed in experiments on NV centers [18], Rydberg atom [20], and high-spin atoms in optical lattices [21].

(ii) When the total magnetization saturates at finite value at long time, the total magnetization oscillates in time. This feature has been observed in experiments using cold molecules in optical lattices [16,17], and fermions in harmonic traps [19].

Here we will address the question of the general conditions under which these two features will occur in these random spin models. This paper is to show that a scenario emerged from the dual gravity theory unifies these experiments.

This paper establishes a connection between the quench dynamics in these random spin models and the dynamics after suddenly turning off the coupling in a traversable wormhole. The connection is bridged by a model coupling two SYK systems proposed by Maldacena and Qi, as shown in Fig. 1(a).
The Maldacena-Qi model is dual to a gravity theory hosting an eternal traversable wormhole [6], and a clear correspondence can be established between the behaviors of the spectral function in different phases of the Maldacena-Qi model and different space-time geometry in the gravity side with or without a traversable wormhole. We will show that the random spin models are closely related to the Maldacena-Qi model, and the spectral functions of these two models share similar behaviors. Hence, by studying the evolution of the spectral functions during the quench dynamics, we can establish a gravity interpretation of the quench dynamics.

\section{MALDACENA-QI MODEL AND ITS GRAVITY DUAL}

Since the Maldacena-Qi model is a coupled SYK model, we need to first briefly introduce the SYK model. A single SYK model is written as

$$
\hat{H}_{\mathrm{SYK}}\left(\left\{\psi_{i}\right\}\right)=i^{2} \sum_{1 \leqslant i<j<k<l \leqslant N} J_{i j k l} \hat{\psi}_{i} \hat{\psi}_{j} \hat{\psi}_{k} \hat{\psi}_{l},
$$

where $\hat{\psi}_{i}(i=1, \ldots, N)$ are $N$ Majorana fermion operators, and $J_{i j k l}$ are random Gaussian variables with zero mean and the variance $\left\langle J_{i j k l}^{2}\right\rangle=3 ! J^{2} / N^{3}$. The SYK model is exactly solvable in the large- $N$ limit. It can be shown explicitly that the low-energy physics of the SYK model has an emergent conformal symmetry, which can be dual to the JackiwTeitelboim gravity in the $\mathrm{AdS}_{2}$ geometry with a black hole.

Various extensions of the SYK models have been considered, especially coupled SYK models [6,24-37]. Here we focus on the version proposed by Maldacena and Qi [6]. The 
Maldacena-Qi model is written as

$$
\hat{H}_{\mathrm{MQ}}=\hat{H}_{\mathrm{SYK}}\left(\left\{\hat{\psi}_{i}^{L}\right\}\right)+\hat{H}_{\mathrm{SYK}}\left(\left\{\hat{\psi}_{i}^{R}\right\}\right)+i \mu \sum_{i} \hat{\psi}_{i}^{\mathrm{L}} \hat{\psi}_{i}^{\mathrm{R}} .
$$

where $\hat{\psi}_{i}^{\mathrm{L}}$ and $\hat{\psi}_{i}^{\mathrm{R}}$ denote the left and the right Majorana fermions. If $\mu=0$, there is no coupling between two SYK models, and on the gravity side, two $\mathrm{AdS}_{2}$ spaces are also independent. Therefore, the geodesic starting from the boundary of one $\mathrm{AdS}_{2}$ space can never reach the boundary of the other $\mathrm{AdS}_{2}$ space. The $\mu$ term adds coupling between two boundaries of the $\mathrm{AdS}_{2}$ spaces, which distorts the boundary geometries. At finite temperature, when the distortion is weak, two boundaries still cannot be connected by a geodesic and this is referred to as the "black hole" (BH) phase. However, when the distortion is strong enough, the geodesic starting from the boundary of one $\mathrm{AdS}_{2}$ space can reach the boundary of the other $\mathrm{AdS}_{2}$ space, which renders these two black holes into a traversable wormhole. This is referred to as the "traversable wormhole" (TW) phase. The difference between these two geometries is represented by two different Penrose diagrams in Figs. 1(b) and 1(c).

In the presence of the holographic duality, the behavior of correlation functions are closely related to the connectivity of the space-time geometry through the standard holographic dictionary. Let us consider the lesser Green's function $G_{a a^{\prime}}^{<}(t)=i \sum_{l}\left\langle\hat{\psi}_{l}^{a^{\prime}}(t) \hat{\psi}_{l}^{a}(0)\right\rangle / N$, where $a, a^{\prime}=\mathrm{L}, \mathrm{R}$. Roughly speaking, this Green's function can be physically interpreted as follows: First, a signal is created at $t=0$ by an operator at boundary $a^{\prime}$, and then the signal propagates following the geodesic, and finally, the signal is detected at time $t$ on boundary $a$. In the BH geometry, since there is no connection between two boundaries, it is natural to have $G_{a a^{\prime}}^{<}(t)=0$ for $a \neq a^{\prime}$. For $a=a^{\prime}, G_{a a^{\prime}}^{<}(t)$ decreases as $t$ increases, because as one can see from the Penrose diagram in Fig. 1(b), the geodesic, denoted by the black dotted line, moves away from the boundary. However, the situation is different for the TW geometry. In the TW geometry, as also shown by the Penrose diagram in Fig. 1(c), the geodesic starting from the left boundary can reach the right boundary and can be further bounced back and forth between two boundaries for an eternal traversable wormhole [6]. Thus all $G_{a a^{\prime}}^{<}(t)$ are always finite for $a=a^{\prime}$ and $a \neq a^{\prime}$. At a time when the geodesic arrives at one of the boundaries, the corresponding Green's function reaches a maximum. Therefore, $G_{a a^{\prime}}^{<}(t)$ shows an oscillatory behavior in time. These two different behaviors of $G_{a a^{\prime}}^{<}(t)$ are also illustrated in Figs. 1(b) and 1(c). Similar behaviors can also be found in the quantity $i \sum_{l}\left\langle\left[\hat{\psi}_{l}^{a}(0), \hat{\psi}_{l}^{a^{\prime}}(t)\right]\right\rangle / N=$ $-G_{a a^{\prime}}^{>}(t)-G_{a a^{\prime}}^{<}(t)$, which more directly reflects whether and how the information gets transferred from one side to the other.

Knowing the Green's function $G_{a a^{\prime}}^{<}(t)$, one can obtain $G_{a a^{\prime}}^{<}(\omega)$ by performing the Fourier transformation, and then derive the spectral function $\rho_{a a^{\prime}}(\omega)=G_{a a^{\prime}}^{<}(\omega) / 2 \pi i n_{F}(\omega)$ by the fluctuation-dissipation theorem, where $n_{F}(\omega)$ is the FermiDirac distribution. In the $\mathrm{BH}$ phase, $\rho_{a a^{\prime}}(\omega)$ shows a single broad peak near $\omega \sim 0$ for $a=a^{\prime}$, and is significantly smaller for $a \neq a^{\prime}$. In the TW phase, $\rho_{a a^{\prime}}(\omega)$ displays multiple narrow peaks. $\rho_{a a^{\prime}}(\omega)$ behaves similarly for $a=a^{\prime}$ and $a \neq a^{\prime}$, except for their different parities in $\omega$. This difference is also shown in Figs. 1(b) and 1(c). Hence, we have established correspondence between the behavior of the spectral function and the presence of a traversable wormhole. Equipped with this correspondence, we are now ready to move on to discuss the random spin models.

\section{CONNECTION BETWEEN THE RANDOM SPIN MODELS AND THE MALDACENA-QI MODEL}

When $\Delta=1, h=0$, and $\bar{J}=0$, the model Eq. (1) possesses $S U(2)$ symmetry, which becomes the Sachdev-Ye model when the $S U(2)$ group is promoted to $S U(M)$ group. The Sachdev-Ye model can be solved in the large- $N$ and large- $M$ limits where the self-energies can be obtained by summing up the melon diagrams only. Here we will take similar approximations for studying the quench dynamics of the model Eq. (1) in general, and the approximations can be justified by qualitative agreements with exact diagonalizations [38] and experiments. Before studying the quench dynamics, we first address the connection between this model and the Maldacena-Qi model.

First of all, the random spin model can be written in terms of fermion operators $\hat{c}_{i, s}$ with spins $s=\uparrow, \downarrow$, by rewriting the spin operators as $\hat{S}_{i}^{\alpha}=\frac{1}{2} \hat{c}_{i, s}^{\dagger}\left(\sigma^{\alpha}\right)_{s s^{\prime}} \hat{c}_{i, s^{\prime}}$, where $\alpha=x, y, z$ and $\sigma^{\alpha}$ denotes the corresponding Pauli matrices. Then, we define the imaginary-time Green's function of fermions $G(\tau)$ as a $2 \times 2$ matrix, with the matrix elements defined as

$$
G_{s s^{\prime}}(\tau)=\frac{1}{N} \sum_{i}\left\langle\mathcal{T}_{\tau} \hat{c}_{i, s}(\tau) \hat{c}_{i, s^{\prime}}^{\dagger}(0)\right\rangle
$$

We first consider the case with $\bar{J}=0$. The Fourier transformation of $G(\tau)$ defines $G\left(i \omega_{n}\right)$, and by the Schwinger-Dyson equation, we have

$$
G\left(i \omega_{n}\right)^{-1}=-i \omega_{n}-\frac{h}{2} \sigma^{x}-\Sigma\left(i \omega_{n}\right),
$$

with the Matsubara frequency $\omega_{n}=(2 n+1) \pi / \beta$ and $\beta=$ $1 /\left(k_{\mathrm{B}} T\right)$ being the inverse temperature, where $\Sigma\left(i \omega_{n}\right)$ is the self-energy in the Matsubara frequency domain. With the large $N$ and the melon diagram approximations, the selfenergy can be obtained as

$$
\Sigma(\tau)=-\frac{J^{2}}{4} \sum_{\alpha \alpha^{\prime}} \xi^{\alpha} \xi^{\alpha^{\prime}} \sigma^{\alpha^{\prime}} G(\tau) \sigma^{\alpha} \operatorname{Tr}\left[\sigma^{\alpha^{\prime}} G(\tau) \sigma^{\alpha} G(-\tau)\right],
$$

where $\xi=(1,1, \Delta)$.

For the Maldacena-Qi model, we can similarly define a $2 \times 2$ matrix $G(\tau)$ with $G_{a a^{\prime}}(\tau)=\sum_{i}\left\langle\mathcal{T}_{\tau} \hat{\psi}_{i}^{a}(\tau) \hat{\psi}_{i}^{a^{\prime}}(0)\right\rangle / N$, with $a, a^{\prime}=\mathrm{L}, \mathrm{R}$, and $G\left(i \omega_{n}\right)$ in the Matsubara frequency domain also obeys the Schwinger-Dyson equation as

$$
G\left(i \omega_{n}\right)^{-1}=-i \omega_{n}-\mu \sigma^{y}-\Sigma\left(i \omega_{n}\right) .
$$

It has been shown that the Green's function obeys the selfconsistency equation [6]

$$
\Sigma_{a a^{\prime}}(\tau)=J^{2} G_{a a^{\prime}}(\tau)^{3} .
$$



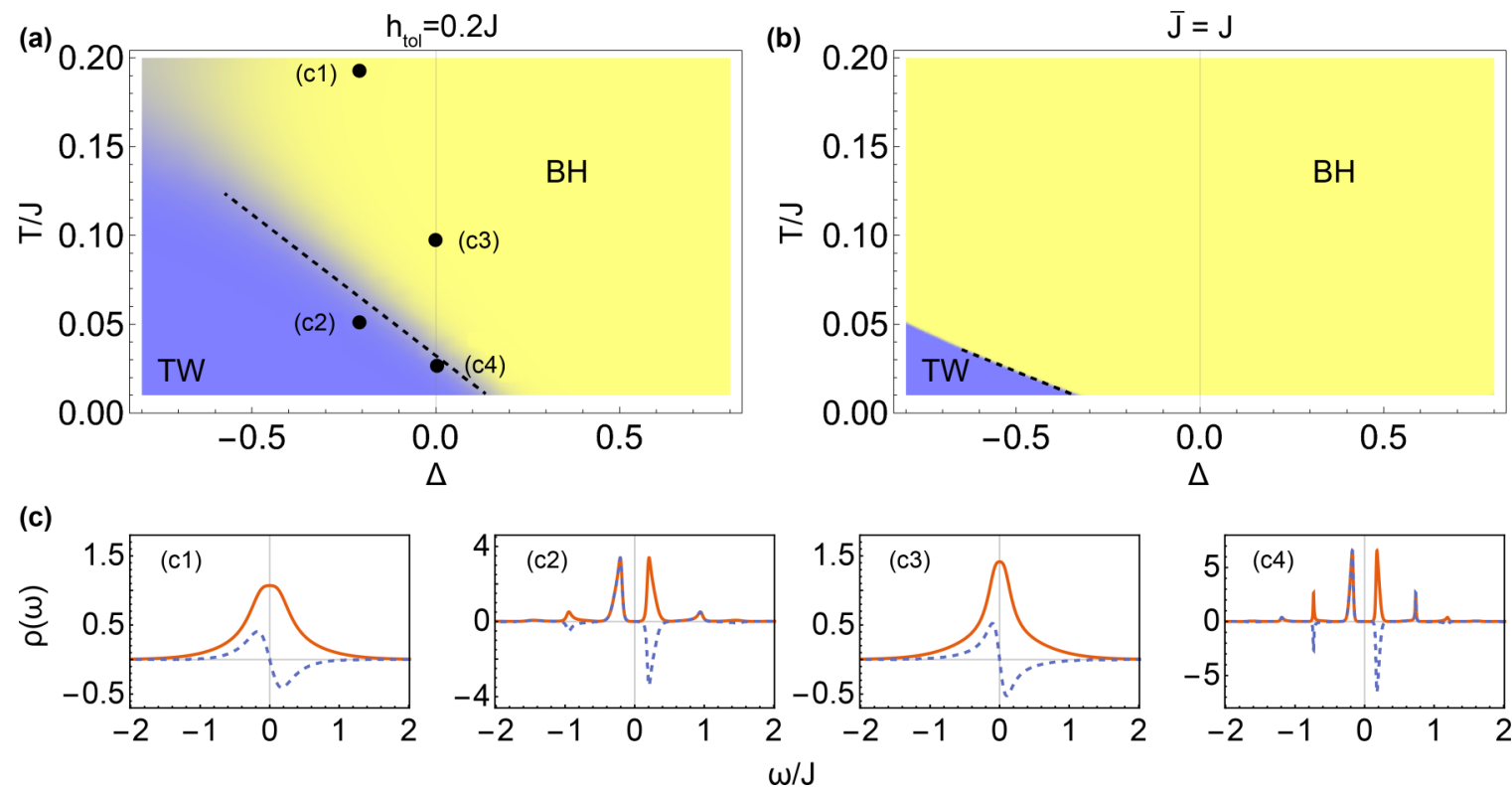

FIG. 2. Phase diagrams of the Hamiltonian before and after quench. (a) Phase diagram for Hamiltonian before quench. Here $h \neq 0$ and $h_{\text {tol }}$ determines the spectral functions, and we set $h_{\text {tol }}=0.2 J$. (b) Phase diagram for Hamiltonian after a quench. Here $h=0$ and $\bar{J}$ is the only parameter left, and we set $\bar{J}=J . J$ is taken as the energy unit. "BH" denotes the black hole phase and "TW" denotes the traversable wormhole phase. They are distinguished by the spectral functions, as illustrated in Fig. 1. The dashed line represents a first-order transition separating two phases, associated with discontinuity in observables, and it is a crossover between two phases for other places. (c) Several typical examples of the spectral functions of $\mathrm{BH}$ and TW phases are plotted, with their corresponding locations marked by solid circles in (a).

We first consider the case $\Delta=0$, where direct evaluation of Eq. (6) yields

$$
\begin{aligned}
& \Sigma_{s s}(\tau)=-J^{2} G_{s s}(\tau) G_{\bar{S} \bar{s}}(\tau) G_{\bar{s} \bar{s}}(-\tau), \\
& \Sigma_{s \bar{s}}(\tau)=-J^{2} G_{s \bar{s}}(\tau) G_{\bar{s} s}(\tau) G_{s \bar{s}}(-\tau),
\end{aligned}
$$

where $\bar{s} \neq s$. First of all, to compare Eq. (5) with Eq. (7), we notice that the spin model has rotational symmetry along $\hat{z}$, and therefore one can replace $\sigma^{x}$ in Eq. (5) by $\sigma^{y}$. Then note that the system is invariant when rotating $\pi$ along $\hat{y}: \hat{c}_{i} \rightarrow$ $i \sigma_{y} \hat{c}_{i}$, which gives $G_{\uparrow \uparrow}(\tau)=G_{\downarrow \downarrow}(\tau)$ and $G_{\uparrow \downarrow}(\tau)=-G_{\downarrow \uparrow}(\tau)$, and the system also has the particle-hole symmetry $\hat{c}_{i} \rightarrow$ $\hat{c}_{i}^{\dagger}$ that corresponds to $\left(\hat{S}_{i}^{x}, \hat{S}_{i}^{y}, \hat{S}_{i}^{z}\right) \rightarrow\left(-\hat{S}_{i}^{x}, \hat{S}_{i}^{y},-\hat{S}_{i}^{z}\right)$, which gives $G_{\uparrow \uparrow}(\tau)=-G_{\uparrow \uparrow}(-\tau)$ and $G_{\uparrow \downarrow}(\tau)=-G_{\downarrow \uparrow}(-\tau)$. These symmetry relations simplify Eq. (9) into $\Sigma_{s s^{\prime}}(\tau)=J^{2} G_{s s^{\prime}}(\tau)^{3}$, which takes the same form as Eq. (8). In this way, Eq. (5) and Eq. (9) for the random spin model become completely identical to Eq. (7) and Eq. (8) for the Maldacena-Qi model. Two spin components $s, s^{\prime}=\uparrow, \downarrow$ play the role of $a, a^{\prime}=\mathrm{L}, \mathrm{R}$, and the Zeeman field $h$ plays the role of the coupling term $\mu$, which is responsible for the wormhole being traversable. The equivalence between these two models also holds approximately for nonzero but small $\Delta$, where a nonzero small $\Delta$ provides an extra channel to couple two sides, as one can see from the low energy effective action [38].

Next, we discuss how to deal with a nonzero $\bar{J}$. Up to a constant, the term associated with $\bar{J}$ can be cast into

$$
\frac{\bar{J}}{2 N} \sum_{\alpha=x, y, z} \xi^{\alpha}\left(\sum_{i} \hat{S}_{i}^{\alpha}\right)\left(\sum_{i} \hat{S}_{i}^{\alpha}\right) .
$$

We implement the molecular field approximation by denoting $M^{\alpha}=\sum_{i}\left\langle\hat{S}_{i}^{\alpha}\right\rangle / N$ and $h_{\text {eff }}^{\alpha}=\bar{J} \xi^{\alpha} M^{\alpha}$. Then, Eq. (10) can be written as

$$
\sum_{\alpha=x, y, z} h_{\mathrm{eff}}^{\alpha} \sum_{i} \hat{S}_{i}^{\alpha}
$$

For equilibrium, $M^{\alpha}$ needs to be determined self-consistently. For dynamics, $M^{\alpha}$ evolves in time. In this paper, since we initially polarize spins along $\hat{x}$, we focus on the situation only $M^{x} \neq 0$ and $M^{y}=M^{z}=0$. Hence, this term can be combined with the $h$ term by replacing $h$ with $h_{\text {tol }}=h-h_{\text {eff }}^{x}=$ $h-\bar{J} M^{x}$.

\section{PHASE DIAGRAM}

The quenching process we will consider is instantaneously turning off $h$ from nonzero to zero, therefore, here we will first discuss the equilibrium phase diagram for both finite and zero $h$, respectively. The model parameters contain $J, \Delta, h$, and $\bar{J}$, as well as temperature $T$. Here we take $J$ as the energy unit. When $h$ is finite, it polarizes spins, and $M^{x}$ is nonzero, which further normalizes $h_{\text {tol }}$ through the effect of $\bar{J}$. When the self-consistency is reached, there is a one-to-one correspondence between $h$ and $h_{\text {tol }}$, and only $h_{\text {tol }}$ enters the equation that determines the spectral functions. Hence, in Fig. 2(a), we fix $h_{\text {tol }}$ and plot the phase diagram in terms of the other two dimensionless parameters $T / J$ and $\Delta$. The phase diagram is obtained by numerically solving the self-consistency equations [38]. Here the "BH" and "TW" are distinguished by their different behaviors of spectral functions, as we illustrate in Figs. 1(b) and 1(c) and show in Fig. 2(c). Consistent with the Maldacena-Qi model [6], the random spin model with small $\Delta$ 
(a)

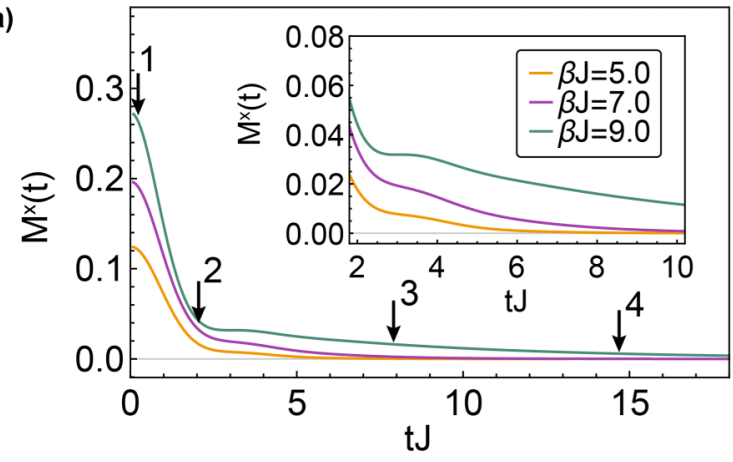

(b)

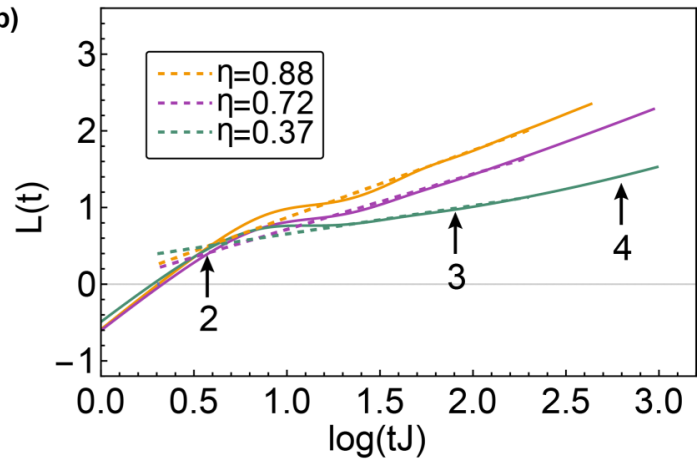

(c)

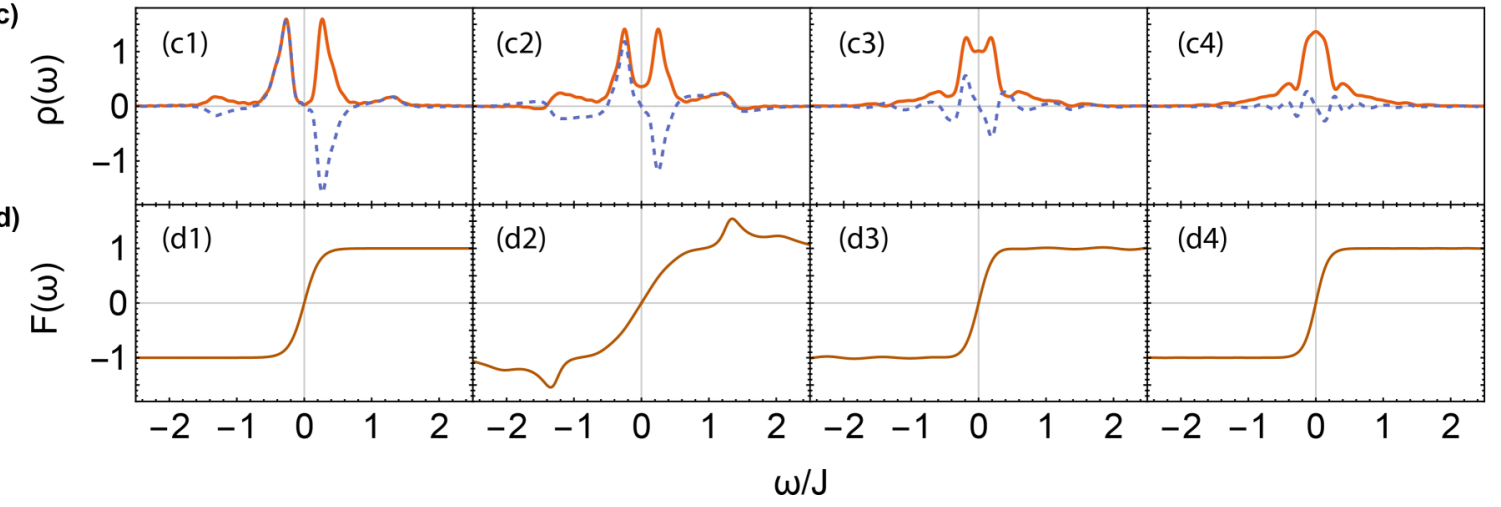

FIG. 3. Slow dynamics after quench. (a) The decay of the total magnetization $M^{x}$ along $\hat{x}$ as a function of time $t$ (in unit of $1 / J$ ) for three different temperatures $\beta J$. The inset shows a zoom-in plot for $t J>t^{*} J \sim 2$. (b) The same curves as (a), but the loglog-log plot is used. Here the vertical axes $\mathcal{L}(t)$ denotes $\log \left(\left|\log \left(M^{x}(t) / M^{x}(0)\right)\right|\right)$. The slope of $\mathcal{L}-\log (t J)$ curves give the exponent in the stretched exponentials. (c) Four representative spectral functions $\rho(\omega)$ and distribution functions $\mathcal{F}(\omega)$ for different stages of the evolution, as marked in (a) and (b). Here we take $\Delta=-0.73$, which is realized in the Rydberg atom experiment [20], and we set $\bar{J}=J$.

displays the $\mathrm{BH}$ phase at high temperature and the TW phase at low temperature [38].

When $h=0$, the system possesses a spin-rotation symmetry of $\left(\hat{S}_{i}^{x}, \hat{S}_{i}^{y}, \hat{S}_{i}^{z}\right) \rightarrow\left(-\hat{S}_{i}^{x},-\hat{S}_{i}^{y}, \hat{S}_{i}^{z}\right)$. If this symmetry is respected, then $M^{x}$ is always zero and $h_{\text {tol }}$ is also zero. Nevertheless, the ground state can have nonzero $M^{x}$ due to the spontaneous symmetry breaking, which leads to nonzero $h_{\text {tol }}$. Both a nonzero $\bar{J}$ and a nonzero $\Delta$ can lead the system into a TW phase $[33,34]$. However, as shown in Fig. 2(b), the TW phase is significantly smaller compared with the $h \neq 0$ case shown in Fig. 2(a).

\section{QUENCH DYNAMICS}

Now we discuss the two universal features observed in the quench experiments. As we have seen, the $h$ term is mapped to the $\mu$ term in the Maldacena-Qi model, which couples two boundaries and makes the wormhole traversable. Hence, in the gravity picture, the quench dynamics of suddenly turning off $h$ corresponds to turning off the coupling term, which is responsible for making a wormhole traversable. Below we will calculate the quench dynamics numerically by the KadanoffBaym formula, which gives how the spectral function evolves in time [38]. By the correspondence between the spectral function and the space-time geometry discussed above, we can establish a gravity picture of the quench dynamics.

Firstly, we discuss the slow dynamics described by a stretched exponential. Initially, the spectral function is a typical TW-type one, as shown in Fig. 3(c1). We show in Fig. 3(a) that the dynamics contain two stages. In the first stage when $t J<t^{*} J$, with $t^{*} J \approx 2$ in the case shown in Fig. 3(a), $M^{x}$ decays quite fast. As shown in Fig. 3(c2), the spectral function still retains TW-phase-like in this stage. It is known that the distribution function $\mathcal{F}(\omega)$ at equilibrium should be $\tanh (\beta \omega / 2)$, as in the initial case shown in Fig. 3(d1). As one can see in Fig. 3(d2), at the end of the first stage, the system strongly deviates from equilibrium.

In the second stage, with $t J$ ranging from $t^{*} J$ to a few tens, the dynamics is much slower. Note that if $M^{x}$ obeys a stretched exponential $e^{-C\left(t / t_{0}\right)^{\eta}}$, it should manifest as a straight line in the $\log \left(\left|\log M^{x}\right|\right)-\log t$ plot, and the slope determines $\eta$. We show this in Fig. 3(b), and the fitting yields $\eta<1$, meaning that the dynamics is slower than usual exponential decay. In Fig. 3(c3), we show that the multiple peaks in the spectral function gradually merge into a single peak, and meanwhile, $\rho_{s s^{\prime}}$ with $s \neq s^{\prime}$ is gradually suppressed. At the long-time limit, as shown in Fig. 3(c4), the spectral function becomes BH-phase-like, where $\rho_{s s^{\prime}}$ shows a single broad peak around $\omega \sim 0$ for $s=s^{\prime}$ and is vanishing small for $s \neq s^{\prime}$. Hence, in the gravity picture, this process corresponds to the wormhole gradually closing. From Figs. 3(d3) and 3(d4), we also see that the distribution functions fast reach quasi-equilibrium in this stage, and by fitting the distribution function with $\tanh (\tilde{\beta} \omega / 2)$, we can obtain an effective temperature $1 / \tilde{\beta}$ for the saturated state. By comparing Fig. 3(d4) with Fig. 3(d1), one can see that the temperature for the final state is close 

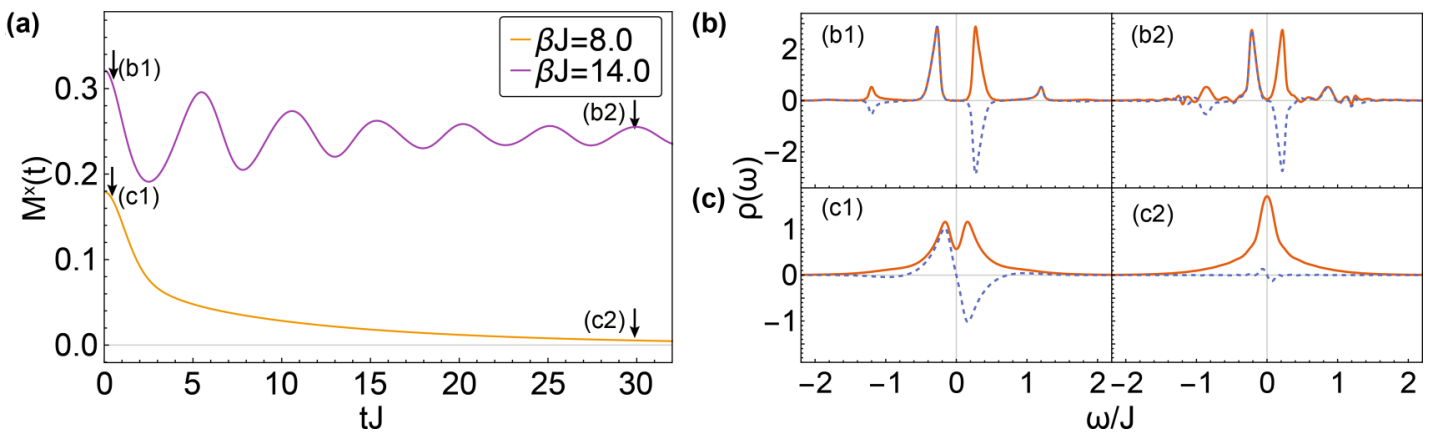

FIG. 4. Oscillatory behavior after quench. (a) The decay of the total magnetization $M^{x}$ along $\hat{x}$ as a function of time $t$ (in the unit of $1 / J$ ) for two different temperatures $\beta J$. (b) The spectral functions $\rho(\omega)$ at the initial time of these two cases [(b1) and (c1)], and at the long-time of these two cases [(b2) and (c2)]. Here we have taken $\Delta=-0.5$ and $\bar{J}=0$.

to the temperature of the initial state. By examining various different parameters [38], we identify that the stretched exponential decay occurs generically when a traversable wormhole is disconnected and becomes two decoupled black holes, and the temperatures of the initial TW and the final $\mathrm{BH}$ phases are close.

Secondly, we discuss the oscillatory behavior. In Fig. 4 we compare two cases, which only differ by the parameter $\beta J$ but show two different behaviors. For the one with a smaller $\beta J, M^{x}$ monotonically decays to zero as discussed above. For the one with a larger $\beta J, M^{x}$ saturates to a finite value in the long-time limit, and oscillates in time before saturation. Here we also want to find a generic condition to determine which behavior should take place, and it turns out that the answer is also quite clear in the gravity picture. As we show in Figs. 4(b) and 4(c), these two cases have similar initial states in the TW phase. However, their long-time saturation states are very different. $\rho(\omega)$ at long-time exhibits typical behavior of the $\mathrm{BH}$ phase for the one with smaller $\beta J$, but exhibits typical behavior of the TW phase for the one with larger $\beta J$. Note that in Fig. 2(b), even with $h=0$, there still exists TW phase in the equilibrium phase diagram, which means that turning off $h$ does not always disconnect the wormhole. If the long-time saturation phase is still retained in the TW phase, there will exhibit an oscillatory behavior of magnetization in time. This oscillatory behavior can also be found by using the effective action with gravity dual [38]. By examining different parameters [38], we also find that whether the monotonical decay or oscillatory behavior occurs in the quench dynamics essentially depends on whether the final state is the BH phase or the TW phase.

\section{SUMMARY AND OUTLOOK}

In summary, we show that the quench dynamics of randomly interacting quantum spins after turning off an external field can be understood in the dual gravity picture as turning off a coupling field for making the wormhole traversable. If this process disconnects the traversable wormhole and finally yields two decoupled black holes, the magnetization displays a slow decay described by the stretched exponential function toward zero magnetization. If this process does not disconnect the traversable wormhole, due to the existence of the residual couplings between the boundary fields, the magnetization saturates to a nonzero value at long time and oscillates around the saturation value. This gravity picture conceptually unifies these two universal phenomena observed in the random spin model realized in different physical systems.

The stratagem of our paper is reminiscent of the concept of the "fixed point" in the renormalization group theory, where different microscopic models can share the same low-energy description given by a fixed point action. Here we use the Maldacena-Qi model, whose gravity dual has been shown explicitly, as the "holographic fixed point", and we use the holographic fixed point to define correspondence between quantum properties and the gravity properties. We further share this correspondence with different microscopic models in the neighborhood of the Maldacena-Qi model with similar quantum properties, and these models are much closer to reality. Therefore, the success of this approach can inspire more applications on realistic quantum models with insights from the gravity theory.

\section{ACKNOWLEDGMENTS}

We thank Yiming Chen, Bartek Czech, Ruihua Fan, Yingfei Gu, Chao-Ming Jian, Xinhua Peng, Xiao-Liang Qi and Yi-Zhuang You for helpful discussions. This work is supported by Beijing Outstanding Young Scientist Program (H.Z.), NSFC Grant No. 11734010 (H.Z. and Y.C.), NSFC under Grant No. 11604225 (Y.C.), MOST under Grant No. 2016YFA0301600 (H.Z.) and Beijing Natural Science Foundation (Grant No. Z180013) (Y.C.).
[1] D. T. Son and A. O. Starinets, Viscosity, black holes, and quantum field theory, Annu. Rev. Nucl. Part. Sci. 57, 95 (2007).

[2] J. Zaanen, Y. Liu, Y.-W. Sun, and K. Schalm, Holographic Duality in Condensed Matter Physics (Cambridge University Press, Cambridge, 2015).
[3] S. A. Hartnoll, A. Lucas, and S. Sachdev, Holographic Quantum Matter (MIT Press, Cambridge, 2018).

[4] P. Gao, D. L. Jafferis, and A. C. Wall, Traversable wormholes via a double trace deformation, J. High Energy Phys. 12 (2017) 151. 
[5] J. Maldacena, D. Stanford, and Z. Yang, Diving into traversable wormholes, Fortschr. der Phys. 65, 1700034 (2017).

[6] J. Maldacena and X.-L. Qi, Eternal traversable wormhole, arXiv:1804.00491.

[7] S. Sachdev and J. Ye, Gapless Spin-Fluid Ground State in a Random Quantum Heisenberg Magnet, Phys. Rev. Lett. 70, 3339 (1993).

[8] A. Kitaev, Talk Given at the KITP Program: Entanglement in Strongly-Correlated Quantum Matter, KITP Program (2015).

[9] J. Maldacena and D. Stanford, Remarks on the Sachdev-YeKitaev model, Phys. Rev. D 94, 106002 (2016).

[10] A. Kitaev and S. J. Suh, The soft mode in the Sachdev-YeKitaev model and its gravity dual, J. High Energy Phys. 05 (2018) 183

[11] P. K. Kovtun, D. T. Son, and A. O. Starinets, Viscosity in Strongly Interacting Quantum Field Theories from Black Hole Physics, Phys. Rev. Lett. 94, 111601 (2005).

[12] C. Cao, E. Elliott, J. Joseph, H. Wu, J. Petricka, T. Schäfer, and J. E. Thomas, Universal quantum viscosity in a unitary Fermi gas, Science 331, 58 (2011).

[13] T. Hartman, S. A. Hartnoll, and R. Mahajan, Upper Bound on Diffusivity, Phys. Rev. Lett. 119, 141601 (2017).

[14] S. A. Hartnoll, Theory of universal incoherent metallic transport, Nat. Phys. 11, 54 (2015).

[15] M. Blake, Universal Charge Diffusion and the Butterfly Effect in Holographic Theories, Phys. Rev. Lett. 117, 091601 (2016).

[16] B. Yan, S. A. Moses, B. Gadway, J. P. Covey, K. R. Hazzard, A. M. Rey, D. S. Jin, and J. Ye, Observation of dipolar spinexchange interactions with lattice-confined polar molecules, Nature (London) 501, 521 (2013).

[17] K. R. A. Hazzard, B. Gadway, M. Foss-Feig, B. Yan, S. A. Moses, J. P. Covey, N. Y. Yao, M. D. Lukin, J. Ye, D. S. Jin, and A. M. Rey, Many-Body Dynamics of Dipolar Molecules in an Optical Lattice, Phys. Rev. Lett. 113, 195302 (2014).

[18] G. Kucsko, S. Choi, J. Choi, P. C. Maurer, H. Zhou, R. Landig, H. Sumiya, S. Onoda, J. Isoya, F. Jelezko et al., Critical Thermalization of a Disordered Dipolar Spin System in Diamond, Phys. Rev. Lett. 121, 023601 (2018).

[19] S. Smale, P. He, B. A. Olsen, K. G. Jackson, H. Sharum, S. Trotzky, J. Marino, A. M. Rey, and J. H. Thywissen, Observation of a transition between dynamical phases in a quantum degenerate Fermi gas, Sci. Adv. 5, eaax1568 (2019).

[20] A. Signoles, T. Franz, R. F. Alves, M. Gärttner, S. Whitlock, G. Zürn, and M. Weidemüller, Glassy Dynamics in a Disordered Quantum Spin System, Phys. Rev. X 11, 011011 (2021).

[21] L. Gabardos, B. Zhu, S. Lepoutre, A. M. Rey, B. LaburtheTolra, and L. Vernac, Relaxation of the Collective Magnetization of a Dense 3D Array of Interacting Dipolar $S=3$ Atoms, Phys. Rev. Lett. 125, 143401 (2020).
[22] H. G. Krojanski and D. Suter, Reduced Decoherence in Large Quantum Registers, Phys. Rev. Lett. 97, 150503 (2006).

[23] M. Lovrić, H. G. Krojanski, and D. Suter, Decoherence in large quantum registers under variable interaction with the environment, Phys. Rev. A 75, 042305 (2007).

[24] S. Banerjee and E. Altman, Solvable model for a dynamical quantum phase transition from fast to slow scrambling, Phys. Rev. B 95, 134302 (2017).

[25] Y. Gu, X.-L. Qi, and D. Stanford, Local criticality, diffusion and chaos in generalized Sachdev-Ye-Kitaev models, J. High Energy Phys. 05 (2017) 125.

[26] R. A. Davison, W. Fu, A. Georges, Y. Gu, K. Jensen, and S. Sachdev, Thermoelectric transport in disordered metals without quasiparticles: The Sachdev-Ye-Kitaev models and holography, Phys. Rev. B 95, 155131 (2017).

[27] X. Chen, R. Fan, Y. Chen, H. Zhai, and P. Zhang, Competition Between Chaotic and Nonchaotic Phases in a Quadratically Coupled Sachdev-Ye-Kitaev Model, Phys. Rev. Lett. 119, 207603 (2017).

[28] X.-Y. Song, C.-M. Jian, and L. Balents, Strongly Correlated Metal Built from Sachdev-Ye-Kitaev Models, Phys. Rev. Lett. 119, 216601 (2017).

[29] S.-K. Jian and H. Yao, Solvable Sachdev-Ye-Kitaev Models in Higher Dimensions: From Diffusion to Many-Body Localization, Phys. Rev. Lett. 119, 206602 (2017).

[30] P. Zhang, Dispersive Sachdev-Ye-Kitaev model: Band structure and quantum chaos, Phys. Rev. B 96, 205138 (2017).

[31] C.-M. Jian, Z. Bi, and C. Xu, Model for continuous thermal metal to insulator transition, Phys. Rev. B 96, 115122 (2017).

[32] D. Chowdhury, Y. Werman, E. Berg, and T. Senthil, Translationally Invariant Non-Fermi-Liquid Metals with Critical Fermi Surfaces: Solvable Models, Phys. Rev. X 8, 031024 (2018).

[33] J. Kim, I. R. Klebanov, G. Tarnopolsky, and W. Zhao, Symmetry Breaking in Coupled SYK or Tensor Models, Phys. Rev. X 9, 021043 (2019).

[34] I. R. Klebanov, A. Milekhin, G. Tarnopolsky, and W. Zhao, Spontaneous breaking of $U(1)$ symmetry in coupled complex SYK models, J. High Energy Phys. 11 (2020) 162.

[35] J. Maldacena and A. Milekhin, SYK Wormhole formation in real time, arXiv:1912.03276.

[36] S. Plugge, É. Lantagne-Hurtubise, and M. Franz, Revival Dynamics in a Traversable Wormhole, Phys. Rev. Lett. 124, 221601 (2020).

[37] S. Sahoo, E. Lantagne-Hurtubise, S. Plugge, and M. Franz, Traversable wormhole and Hawking-Page transition in coupled complex SYK models, Phys. Rev. Research 2, 043049 (2020).

[38] See Supplemental Material at http://link.aps.org/supplemental/ 10.1103/PhysRevResearch.3.L022024 for detailed derivations and more numerical examples. 